\title{
Verso il gigantismo delle ASL: implicazioni sulla qualità dell'assistenza e il management
}

\author{
Elio Borgonovi
}

Dopo il periodo dell'aziendalizzazione degli anni '90, del contenimento dei costi non del tutto efficace nel primo decennio del nuovo secolo e diventato molto stringente a partire dal 2010, della razionalizzazione del sistema di offerta con una forte riduzione del numero dei posti letto e dei ricoveri, oggi il SSN sta vivendo la fase di orientamento al gigantismo. L'esperienza dell'azienda unica della Regione Marche è oggi seguita da molte regioni che hanno realizzato o intendono realizzare, con appositi progetti di legge regionale, la riduzione del numero delle ASL e di conseguenza l'aumento delle loro dimensioni medie.

Una tendenza che ha radici lontane nelle teorie sulle economie di dimensione del mondo industriale, rinnovate dall'impatto delle nuove tecnologie il cui impiego richiede elevati volumi per consentire di ammortizzare gli ingenti investimenti. Questa tendenza è stata ulteriormente accentuata dalla competizione globale che, sintetizzando la complessità del problema, si attua sui principi di scalabilità, replicabilità, nicchie. La scalabilità significa appunto il perseguimento di economia tramite l'ampliamento dei mercati di sbocco e quindi delle dimensioni della produzione con abbattimento dei costi unitari. La replicabilità rappresenta in un certo senso l'estensione al "modello di business" del principio di scalabilità. Il vantaggio competitivo si realizza replicando in più contesti lo stesso modello di business, con limitate varianti per i necessari adattamenti a specifiche realtà socioeconomiche, il che consente di ridurre $i$ costi non solo degli investimenti per la produzione, ma in generale quelli connessi all'ideazione, messa a punto e realizzazione di soluzioni vincenti. La ricerca o lo sviluppo di nicchie di mercato è una strategia adottata da imprese innovative (soprattutto per quanto riguarda i prodotti) o da imprese che sfruttano particolari fattori di successo (quali il brand "paese" o di particolari localizzazioni, l'esperienza di gruppi di lavoratori, per esempio artigiani, la flessibilità nella produzione contrapposta alla standardizzazione). La prima strategia è tipica di imprese che aumentano le proprie dimensioni, la seconda è tipica di imprese che si articolano in gruppi di grandi dimensioni presenti in diversi Paesi o in diverse località, la terza è consentita anche a piccole e medie imprese.

Si può dire che nel 1978, al momento della istituzione del Servizio Sanitario Nazionale, la scelta di avere ASL di piccole dimensioni (oltre 600) prefigurava implicitamente o esplicitamente obiettivi di replicabilità (garantire nelle diverse parti del territorio i servizi previsti dai LEA) congiunti a quelli di nicchia (consentire adattamenti a specifiche condizioni socio-economiche e territoriali, per esempio pianura, collina, montagna). Ben presto sono emersi gli effetti negativi della eccessiva frammentazione che hanno indotto tutte le regioni a ridurre il numero portando le ASL sostanzialmente a livello di dimensione provinciale, salvo un certo numero di situazioni di dimensioni sub-provinciali. Con $i$ diversi interventi finalizzati al contenimento del trend di spesa (leggi finanziarie, dal 2009 leggi di stabilità, interventi sui piani di rientro delle regioni in deficit strutturale, spending review a partire dal 2010-2011) l'attenzione è stata posta prevalentemente su due elementi: interventi per ridurre la variabilità dei prezzi negli acquisti di beni e servizi da parte del SSN e riduzione dei costi generali anche tramite la standardizzazione di vari sistemi gestionali quali gestione del personale, contabilità e bilancio, informatica, manutenzioni ecc. Il tema della variabilità dei prezzi è spesso assurto negativamente agli onori della cronaca, in genere collegato a fatti di malasanità, sprechi, corruzione. 
Non sempre i dati riportati erano corretti, per esempio si mettevano a confronto beni e servizi tra loro diversi anche se formalmente rientranti nella stess a "categoria merceologica" o "tipologia" (il classico esempio di cateteri, stent, protesi spesso diversissime tra loro anche se denominate nello stesso modo) o si mettevano a confronto dati raccolti con metodologie molto discutibili (per esempio rapportare le spese di pulizia o di lavanderia al numero di posti letto quando è noto che questi ultimi sono sempre meno un driver di spesa significativo). Nondimeno, chi vive all'interno e conosce il sistema non ha mai negato, né poteva farlo, che sono sempre esistiti spazi (più o meno grandi nelle diverse realtà) di razionalizzazione negli acquisti e nello svolgimento di funzioni che sono replicate in diverse ASL. Già nella seconda metà degli anni '90, e in modo sempre più accentuato nel nuovo secolo, questa consapevolezza è stata alla base di esperienze di acquisti comuni (consorzi guidati da ASL capofila o aree vaste di acquisto variamente denominate). L'orientamento verso un ulteriore aumento delle dimensioni delle ASL viene oggi proposto per perseguire i seguenti obiettivi: ridurre a livello regionale i costi per funzioni generali e comuni (per esempio gestione del personale, sistemi informativi, acquisti ecc.), rafforzare la capacità negoziale delle aziende sanitarie nei rapporti con le aziende fornitrici, consentire una più elevata professionalità nello svolgimento di alcune funzioni (per esempio riduzione delle centrali di acquisto e costituzione di una unica centrale regionale), ridare slancio alla funzione di programmazione e controllo distinguendola in modo più chiaro da quella di erogazione dei servizi. Il primo obiettivo viene perseguito con soluzioni varie, tra le quali si segnalano quella di costituire un'azienda regionale "di servizio" che diventi fornitrice interna e svolga le funzioni per le aziende operative, ossia che hanno la funzione di garantire la tutela della salute tramite l'organizzazione e l'erogazione di servizi. Il secondo obiettivo è, in un certo senso, stimolato dall'evoluzione del contesto esterno. Di fronte a una realtà nella quale vi è una tendenza alla concentrazione delle aziende fornitrici del SSN, si ritiene opportuno un bilanciamento dato dall'aumento di dimensioni delle aziende acquirenti. Potendo negoziare volumi di forniture più elevati è possibile ottenere anche prezzi minori e altre condizioni favorevoli in termini di caratteristiche dei beni forniti, garanzie e assistenza post-vendita, manutenzioni ecc. Il perseguimento di questo obiettivo trova un ulteriore sostegno nella maggiore facilità di collegamento e coordinamento dell'azione di un'unica centrale regionale con la Consip. Per altre funzioni di servizio, per esempio l'impostazione e la gestione di sistemi informativi, è possibile pensare a vantaggi non solo di tipo economico, collegati a una progettazione unitaria, alla inter-operabilità di sistemi che oggi molte volte non si parlano o hanno difficoltà a parlarsi, alla possibilità di controllare la qualità e la significatività dei dati. La funzione di carattere logistico può essere svolta in modi più efficaci ed efficienti quando le soluzioni sono progettate su scala regionale (per regioni fino a due milioni di abitanti) o su grandi aree sub-regionali (per le regioni di più grandi dimensioni). Infatti, anche le società fornitrici di servizi logistici più qualificate hanno dimensioni ampie e preferiscono avere interlocutori di grandi dimensioni. Il terzo obiettivo viene collegato al fatto che, con l'aumento delle dimensioni e la riduzione del numero di ASL, è possibile abbattere i costi di struttura di carattere istituzionale e amministrativo, ma soprattutto attuare processi di standardizzazione e di informatizzazione che riducono il fabbisogno complessivo di personale. Questi processi, a loro volta, consentono da un lato di far fronte ai vincoli sul turnover del personale, senza penalizzare le attività direttamente rivolte ai pazienti, $e$ dall'altro di trovare spazi per adeguare la professionalità degli operatori. Per esempio, invece di avere gruppi limitati di persone dedicate agli acquisti di ogni ASL, è possibile avere gruppi più numerosi di persone che possano specializzarsi nella definizione di bandi per diverse classi o tipologie di acquisti, è possibile dedicare persone alle "ricerche di mercato" sulle aziende fornitrici e non solo alla migliore organizzazione delle procedure di gara, persone dedicate alla comparazione dei costi. ASL di maggiori dimensioni possono trovare spazi per avere persone dedicate alla gestione amministrativa del personale, di paghe e contributi, alla programmazione del personale, all' individuazione dei fabbisogni critici del personale e alla programmazione, alle procedure di reclutamento, formazione e sviluppo professionale. Molti altri esempi potrebbero essere portati per varie funzioni generali e comuni.

Infine, uno degli obiettivi presenti in generale nelle leggi o nelle proposte di legge è quello di consentire una più netta distinzione della funzione di programmazione, acquisto e controllo da quella di produzione ed erogazione dei servizi. In effetti, sia nelle regioni che hanno adottato il modello di integrazione con il mantenimento degli ospedali nell'ambito delle ASL (salvo pochi ospedali di elevata complessità e specializzazione e IRCCS), sia in quelle che hanno adot- 
tato il modello di separazione delle funzioni di acquisto-purchasing (riservato alle aziende sanitarie territoriali) da quelle di erogazione-providing di servizi (riservato alle aziende ospedaliere autonome), vi è stato una specie di "schiacciamento" o centralizzazione della funzione di programmazione e controllo. Tale funzione è stata progressivamente concentrata negli assessorati regionali (con il supporto più o meno coordinato e valorizzato delle agenzie regionali per i servizi sanitari) ed è stata, in un certo senso, atrofizzata nell'ambito delle ASL. Le aree vaste sono rimaste prevalentemente focalizzate sulle problematiche degli acquisti mentre la programmazione delle ASL si è limitata alla dimensione informativo-partecipativa dei comuni (svolta in modo più o meno efficace) prova di reali contenuti programmatori. L'aumento delle dimensioni comporta in genere il ritorno degli ospedali generali per acuti e dei presidi ospedalieri territoriali o di prossimità nell'ambito delle aziende territoriali, per facilitare l'applicazione del principio di continuità dell'assistenza, mentre resterebbero autonomi un numero limitato di ospedali di alta complessità e alta specializzazione (strutture di terzo livello, come si diceva in passato, o hub, come si dice oggi nell'ambito del modello hub and spoke). In questo modo le ASL (che alcune regioni stanno ipotizzando in termini di agenzie sanitarie territoriali, creando non poca confusione terminologica) di più grandi dimensioni (1-2 milioni di abitanti) diventerebbero ambiti o luoghi nei quali la programmazione regionale viene declinata in contesti più omogenei nel proprio interno, mentre all'interno di queste ASL/agenzie si dovrebbero/potrebbero sviluppare ASL/aziende/erogatori di servizi di salute che possono competere tra loro. Le formule ipotizzate sono quelle di aziende di servizi pubbliche, private accreditate, miste pubblico e privato che, tra l'altro, sarebbero spinte anche tramite incentivi o sistemi di finanziamento a orientarsi verso modelli di "presa in carico dei pazienti".

Di fronte a una tendenza cosi consolidata e sostenuta da molti diventa inutile analizzare i prevedibili vantaggi e svantaggi e, soprattutto, appare inutile o velleitario mettere in guardia rispetto a possibili rischi. Appare invece più opportuno e proficuo proporre due domande alle quali cercare di dare risposte convincenti. Sono realistici i vantaggi economici perseguiti? Quali possono essere le conseguenze? Molti rispondono con una certa sicurezza alla prima domanda portando dati sulle "economie di dimensione" ottenuti con gli acquisti centralizzati, su quelli ottenuti in imprese private, su quelli ottenuti in altri Paesi. La prima risposta ha un limite evidente: riguarda solo una funzione e non l'intera gestione. La seconda e la terza risposta richiedono grande cautela poiché si assumono come ripetibili i risultati ottenuti in contesti completamente diversi. Questo limite vale anche quando si propongono stime "cautelative", ossia che propongono economie di dimensioni più ridotte di quelle conseguite in altri contesti. Ma il limite concettuale più rilevante, che poi si traduce in concreto, consiste nel fatto che processi di aggregazione producono economie e diseconomie, queste ultime legate ai costi interni di organizzazioni sempre più complesse e articolate o costi di transazione tra l'azienda unica regionale "di servizio" e le aziende operative. In un sistema caratterizzato da moltissime norme, tra loro non di rado contrastanti (aspetto istituzionale), da dinamiche politiche oggi ancor più frammentate del passato (contesto politico), campanilismi e localismi (aspetto sociale che può essere criticato ma che non si può trascurare), da vincoli e comportamenti sindacali (che per esempio rendono difficile la mobilità), da comportamenti individuali (che non sempre accettano la flessibilità per dare migliori servizi ai pazienti e alla comunità) esiste il concreto rischio che le diseconomie di dimensione superino le economie di dimensione, almeno nel breve e medio periodo.

Per ridurre tale rischio e far prevalere le economie, la transizione verso dimensioni più ampie deve essere definita da normative chiare e semplici sui nuovi assetti istituzionali (mentre i provvedimenti già adottati o in discussione sembrano aggiungere complessità e confusione alla situazione attuale sul piano dei contenuti e non di rado della stessa terminologia). È poi necessario informare e formare le persone con riguardo ai nuovi assetti organizzativi, alle nuove relazioni gerarchiche e funzionali che caratterizzano aziende più ampie, ai sistemi operativi, ai processi decisionali. Infine, cosa che non è presente in nessuna delle leggi regionali già approvate o delle proposte di legge in corso di discussione, è necessario attivare un sistema di monitoraggio e valutazione degli effetti correlati ai nuovi assetti istituzionali e organizzativi, sistemi che devono essere sensibili e operare nel breve periodo. Una delle carenze tipiche del nostro Paese è la mancanza di valutazioni di impatto delle politiche e delle conseguenze correlate ai cambiamenti istituzionali e organizzativi. Applicando la logica della sintomatologia precoce, tipica della sanità e dei "segnali deboli tipici della cultura di management", è necessario capire rapidamente le dinamiche attivate dalla tendenza verso il giganti- 
smo delle aziende sanitarie, poiché se non si interviene con immediatezza per esaltare gli aspetti positivi (economie di dimensione) e bloccare o attenuare gli effetti negativi (diseconomie di dimensione) si corre il rischio che $i$ secondi prevalgano sui primi, si consolidino e diventino difficili da combattere.

Alla domanda relativa alle possibili conseguenze si può rispondere anticipando che, mentre è chiara la distinzione tra acquisto dei fattori produttivi e loro utilizzo nella produzione dei servizi di tutela della salute, quella tra attività di programmazione e controllo, da un lato, e gestione, dall'altro, è chiara solo sul piano teorico e logico, mentre è meno evidente sul piano del concreto operare. Anzi, molte teorie sottolineano che oggi la gestione di aziende complesse non possa prescindere da un'adeguata attività di programmazione (ex ante) e controllo (ex post). Parimenti si sostiene che un'attività di programmazione e controllo che non si traduca in gestione rischia di produrre documenti, buone intenzioni e di restare sulla carta. In effetti, la distinzione ha ragione di esistere solo assumendo il concetto di programmazione e controllo come definizione di regole e monitoraggio-verifica della loro corretta applicazione. Quindi, una distinzione tra programmazione e controllo "di sistema", che resterebbe in carico alle agenzie/aziende di più grandi dimensioni, e programmazione e controllo di gestione, che resterebbe comunque attribuita alle aziende erogatrici di servizi. In questo modo, il modello istituzionale appare astrattamente coerente, ma solleva ulteriori problemi che non possono essere trascurati.

- I vertici delle agenzie/aziende di programmazione e controllo di aree vaste e sub-regionali (comunque li si voglia denominare) devono essere considerati come articolazione territoriale della funzione di politica regionale (rischio di essere considerati o di percepirsi come "assessori di una parte del territorio regionale", come ha affermato recentemente un collega che non cito semplicemente perché non gli ho chiesto l'autorizzazione) o come articolazione territoriale della funzione tecnica del management regionale?

- Comunque la direzione dell'agenzia/azienda di programmazione, acquisto e controllo costituisce un ulteriore livello istituzionale e organizzativo del funzionamento del sistema regionale, con il concreto rischio di rendere ulteriormente complessi i processi decisionali, la formulazione dei budget e la loro traduzione operativa.

- La previsione che all'interno delle aree di competenza delle agenzie/aziende di grandi dimensioni possano operare "aziende di produzione ed erogazione di servizi" (pubbliche, private, miste), in competizione tra loro, appare un lodevole auspicio, un wishful thinking (come dicono gli inglesi) difficile da realizzare in una situazione di carenza strutturale di risorse.

Con riguardo al primo punto, si può essere sicuri che, anche sotto tortura (come si dice in questi casi per rendere più forte l'affermazione), tutti negherebbero che i direttori delle agenzie/aziende sub-regionali possano essere considerati o considerarsi come "assessori di una parte del territorio", ma è altrettanto sicuro il fatto che molti avranno questa tentazione, con la conseguenza che il loro ruolo assumerà una connotazione "politica" o di "politicizzazione", anche perché essi saranno gli interlocutori naturali degli enti locali. Data la frammentazione politica del nostro Paese, $i$ direttori delle agenzie/aziende diventerebbero in qualche modo "interfaccia" o "diaframma" tra i sindaci e i rappresentanti degli enti locali da un lato, e l'assessore/giunta/governatore della Regione dall'altro. Con riferimento all'ipotesi che $i$ direttori delle agenzie/aziende di programmazione acquisto e controllo diventino un'articolazione della "funzione tecnica" di programmazione e controllo della Regione, è appena il caso di ricordare le difficoltà di relazione tra gli assessorati regionali e le agenzie tecniche regionali (che sono state cancellate in alcune regioni) per affermare che sorgeranno altrettante e sicuramente maggiori difficoltà. In entrambi i casi si avrebbe comunque uno schiacciamento e impoverimento della funzione manageriale con un indebolimento della cultura di management che invece appare come esigenza critica del sistema.

L'introduzione di un ulteriore livello istituzionale-organizzativo solleva il problema di precisare tempestivamente $i$ poteri dei diversi organi (chi decide che cosa e chi fa che cosa concretamente), le relazioni di dipendenza funzionale (come ci si deve coordinare ai vari livelli) o gerarchica (che consiste nel chiarire chi decide le carriere delle persone ele loro remunerazioni, e chi decide in caso di pareri contrastanti), a chi viene attribuito concretamente il budget, quali 
criteri di allocazione devono essere adottati, quale sistema di valutazione della performance si intende adottare e a chi è attribuita la gestione di questo sistema.

Con riguardo all'ultima problematica, non bisogna inoltre dimenticare che nel sistema di tutela della salute la logica di introdurre regole in grado di "creare una competizione virtuosa" tra aziende di produzione ed erogazione di servizi "integrati", in grado di garantire la presa in carico di pazienti nella fase acuta, sub-acuta, e di cronicità non può essere considerata assoluta, poiché deve essere bilanciata da una logica altrettanto forte di cooperazione. La logica della competizione può stimolare la ricerca dell'efficienza, della produttività, della riduzione dei costi unitari, ma solo una logica di collaborazione nell'ambito delle agenzie/aziende di programmazione, acquisto e controllo e, più in generale, nell'ambito del sistema regionale, consente di perseguire obiettivi di appropriatezza, efficacia, garanzia uniforme di livelli assistenziali, qualità controllata dei servizi.

Appare evidente che i problemi non mancheranno, perché, come sempre accade, una cosa è progettare, decidere e approvare leggi che riguardano i grandi disegni istituzionali-organizzativi, altra cosa è avere la consapevolezza delle prevedibili implicazioni e sviluppare conoscenze, competenze, capacità per affrontare i temi di attuazione. Si può usare la diffusa affermazione secondo cui "il diavolo (i problemi) si nasconde nei dettagli", ma avendo maturato la consapevolezza dei problemi si può cercare di girare la frase per fare in modo che "le soluzioni positive possano essere trovate da chi sa anticipare i dettagli". 